\title{
Spectral modeling of Fe XVII pumped by a free-electron x-ray laser
}

J. Clementson

July 5,2011

X-Ray Lasers and Coherent X-Ray Sources: Development and Applications

San Diego, CA, United States

August 22, 2011 through August 25, 2011 
This document was prepared as an account of work sponsored by an agency of the United States government. Neither the United States government nor Lawrence Livermore National Security, LLC, nor any of their employees makes any warranty, expressed or implied, or assumes any legal liability or responsibility for the accuracy, completeness, or usefulness of any information, apparatus, product, or process disclosed, or represents that its use would not infringe privately owned rights. Reference herein to any specific commercial product, process, or service by trade name, trademark, manufacturer, or otherwise does not necessarily constitute or imply its endorsement, recommendation, or favoring by the United States government or Lawrence Livermore National Security, LLC. The views and opinions of authors expressed herein do not necessarily state or reflect those of the United States government or Lawrence Livermore National Security, LLC, and shall not be used for advertising or product endorsement purposes. 


\title{
Spectral modeling of Fe XVII pumped by a free-electron x-ray laser
}

\author{
Joel Clementson \\ Lawrence Livermore National Laboratory, Livermore, California 94550, USA
}

\begin{abstract}
The atomic structure and x-ray pumping of neonlike Fe XVII have been calculated and modeled under freeelectron laser excitation conditions using the Flexible Atomic Code. Specifically, pumping of the $\left(2 \mathrm{p}_{3 / 2} 3 \mathrm{~s}_{1 / 2}\right)_{2,1}$, $\left(2 \mathrm{p}_{1 / 2} 3 \mathrm{~s}_{1 / 2}\right)_{1},\left(2 \mathrm{p}_{3 / 2} 3 \mathrm{~d}_{5 / 2}\right)_{1}$, and $\left(2 \mathrm{p}_{1 / 2} 3 \mathrm{~d}_{3 / 2}\right)_{1}$ levels that connect with the ground state $\left(2 \mathrm{~s}^{2} 2 \mathrm{p}^{6}\right)_{0}$ by the socalled M2, 3G, 3F, 3D, and 3C transitions have been studied. In addition, the spectrum of sodiumlike Fe XVI has been modeled to account for possible line coincidences with the neonlike spectrum. The calculations include oscillator strengths, radiative transition probability rates, autoionization rates, non-resonant photoionization cross sections, and line emissivities.
\end{abstract}

Keywords: atomic spectra, x-ray pumping, laboratory astrophysics, free-electron laser

\section{INTRODUCTION}

The combination of electron beam ion traps (EBITs) with accelerator-based light sources is a novel laserspectroscopic technique for the study of x-ray transitions in highly charged ions. The x-ray emission from a synchrotron or free-electron laser (FEL) is guided into an EBIT, where ions of a certain charge state are created and confined in an electromagnetic trap. The laser radiation can then pump specific atomic resonances of the nearly stationary highly charged ions and populate energy levels otherwise not readily excited in a low-density collisional plasma. The technique allows for new regimes of atomic physics to be explored, such as line-formation processes in photoionized plasmas. The EBIT-accelerator arrangement has been tested at laboratories such as the BESSY-II electron storage ring ${ }^{1,2}$ and the FLASH FEL ${ }^{3}$ facilities in Germany. Recently, an EBIT end station was implemented at the new Linac Coherent Light Source (LCLS) at the Stanford SLAC National Accelerator Laboratory $^{4,5}$ in California. The LCLS x-ray free-electron laser (XFEL) is the worlds brightest source of coherent x-rays and can presently vary the x-ray output from $825 \mathrm{eV}$ up to $8250 \mathrm{eV},{ }^{6}$ thereby covering the larger part of the soft x-ray band. With repetition rates of $120 \mathrm{~Hz}$, each laser pulse can deliver around $1 \mathrm{~mJ}$ of energy during a time interval of the order $100 \mathrm{fs}$ to $1 \mathrm{ps}$. This time scale corresponds to typical lifetimes of energy levels in highly charged ions, why excited levels with electric dipole (E1) allowed decay channels actually could be considered semi-metastable since the LCLS beam may excite and ionize trapped ions in multistep processes. Specific population mechanisms of complex spectra could hence be examined and used to benchmark modeling of spectra observed from low-density plasmas with strong radiation fields such as the interstellar medium or plasmas in the vicinity of massive accretion objects. ${ }^{7}$

Iron is one of the most abundant elements in the universe and frequently observed in astrophysical spectra. In order to fully interpret such spectra the atomic radiative and collisional properties need to be known from accurate calculations and laboratory measurements. ${ }^{7}$ The experimental efforts extend from studies of low charge states, such as in the FERRUM project, ${ }^{8,9}$ to investigations of highly charged iron ions found in tokamaks, off laser-irradiated targets, and in EBITs, see e.g. Refs. ${ }^{7,10-15}$ Due to its closed-shell structure neonlike $\mathrm{Fe}^{16+}$ has a high fractional abundance over a wide temperature interval. The Fe XVII spectrum was first measured in 1938 by Tyrén at Uppsala University ${ }^{16}$ and has since been observed numerous times, see e.g. Refs. ${ }^{17-19}$ However, the strengths of the Fe XVII lines have turned out to be problematic as the intensity ratios of some of the strong $n=2-n=3$ lines vary widely between experiments, observations, and spectral modeling. ${ }^{10}$ This has contributed to a very large number of experimental and theoretical investigations be carried out, see e.g. Refs. ${ }^{20-39}$ for some of the more recent works. Depending on plasma properties and available spectral resolution, some line ratios

E-mail: clementson@llnl.gov, Telephone: +1-925-424-4994 
may be explained by satellite line blends, self absorption, or cascade effects. However, there are still features in the Fe XVII spectrum that cannot be satisfactorily treated. The line-formation processes that may contribute to the emission under various conditions need therefore be yet better known from both experiment and theory to allow one of the most observed x-ray spectra in astrophysics to be fully utilized for plasma diagnostics.

Neonlike systems, such as $\mathrm{Fe}^{16+}$, have an energy level structure where the $\left(2 \mathrm{p}_{3 / 2} 3 \mathrm{~s}_{1 / 2}\right)_{2}$ metastable level is the first level above the $\left(2 \mathrm{~s}^{2} 2 \mathrm{p}^{6}\right)_{0}$ ground state and must therefore decay by magnetic quadrupole (M2) transition (neonlike spectra are thus analogous to the closed-shell helium- and nickellike systems, where the lowest excited level decays by a magnetic dipole (M1) and a magnetic octupole (M3) transition, respectively). The transition is commonly labeled $\mathrm{M} 2$ or $3 \mathrm{H}$ and together with seven strong electric dipole (E1) resonance lines, labeled 3A 3G, comprise the lines that are typically the strongest in neonlike spectra, see Fig. 1. The Fe XVII transitions often considered for plasma diagnostics include M2 and the ground-state transitions from $3 \mathrm{G}\left(2 \mathrm{p}_{3 / 2} 3 \mathrm{~s}_{1 / 2}\right)_{1}, 3 \mathrm{~F}$ $\left(2 \mathrm{p}_{1 / 2} 3 \mathrm{~s}_{1 / 2}\right)_{1}, 3 \mathrm{D},\left(2 \mathrm{p}_{3 / 2} 3 \mathrm{~d}_{5 / 2}\right)_{1}$, and $3 \mathrm{C},\left(2 \mathrm{p}_{1 / 2} 3 \mathrm{~d}_{3 / 2}\right)_{1}$. In the following, the line labels will also be used to designate the excited levels.

In this paper, laser excitation of the Fe XVII spectrum is studied. Structure calculations and spectral modeling of the neonlike iron ion have been performed using the $\mathrm{Gu}$ atomic physics code dubbed the Flexible Atomic Code (FAC). ${ }^{40}$ The spectra are modeled for plasma conditions of the LCLS-EBIT setup and focus on x-ray pumping of the diagnostically interesting $\mathrm{M} 2,3 \mathrm{G}, 3 \mathrm{~F}, 3 \mathrm{D}$, and $3 \mathrm{C}$ ground-state transitions. In addition, the spectra of sodiumlike Fe XVI have been calculated to account for possible line coincidences with Fe XVII. The calculations include energy levels, oscillator strengths, non-resonant photoionization cross sections, autoionization rates, and line emissivities.

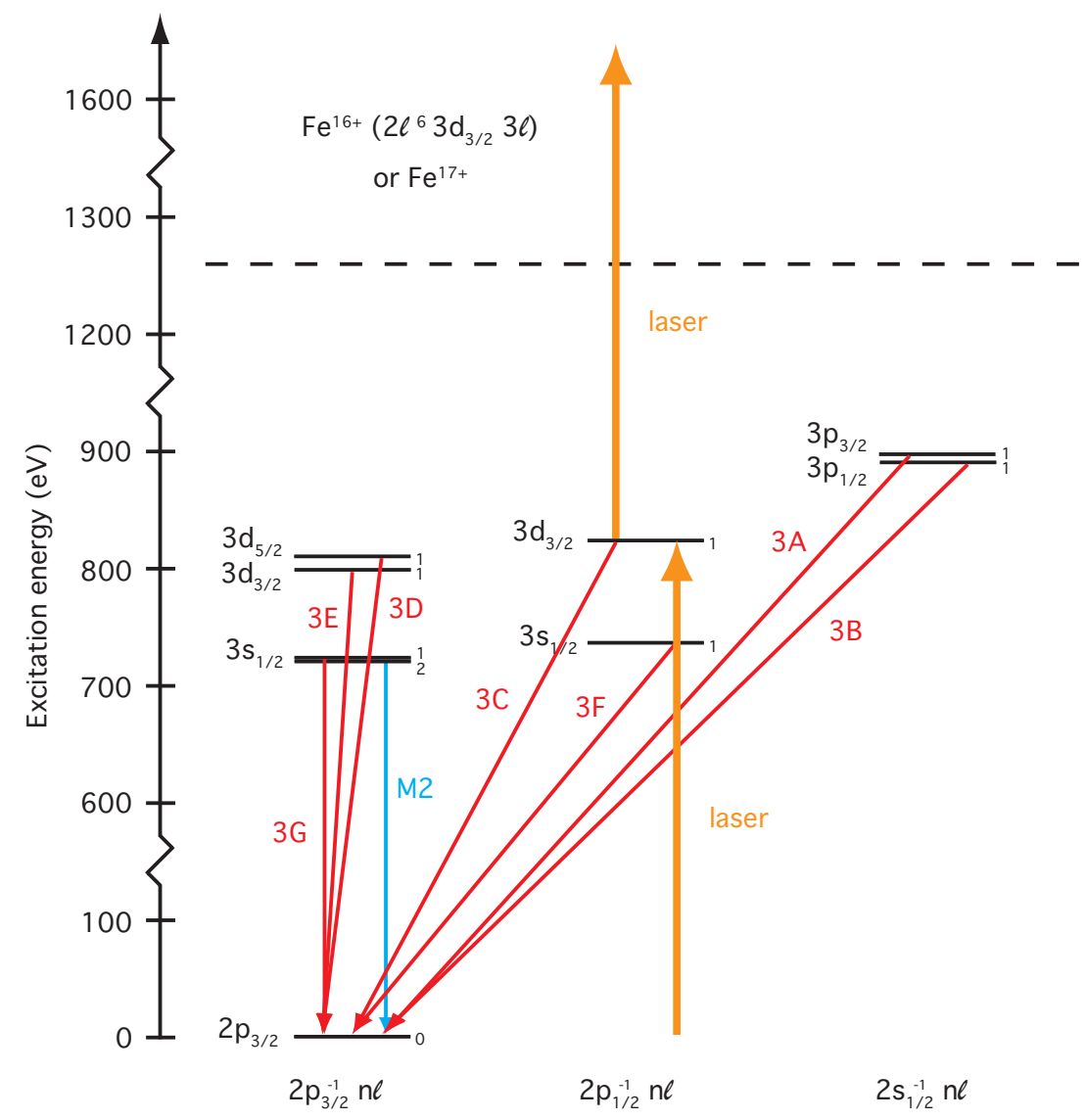

Figure 1. Grotrian diagram of Ne-like $\mathrm{Fe}^{16+}$ displaying the strong $3 \mathrm{~A}-3 \mathrm{G}$ electric dipole transitions and the M2 magnetic quadrupole transition. The yellow transitions indicate the x-ray pumping to and from level $3 \mathrm{C}$ at $825.1 \mathrm{eV}$. See Table 1 for radiative properties. 


\section{CALCULATIONS}

In an EBIT-XFEL plasma the excitation of spectra is dominated by photoabsorption of the ultra-brilliant light from the x-ray laser. The EBIT then only acts as a source and trap for the ions. The coronal equilibrium of the ions is pre-established by electron impact excitation of the beam electrons with the atoms, molecules, or ions that are introduced into the trap, together with recombination and charge exchange processes with beam electrons and the ambient gas. The collisional ionization balance is therefore determined by EBIT parameters such as electron energy, beam current, confinement time, trap voltage potential, and material injection pressure. The electron density $n_{e}$ of the beam in the trap can be estimated from

$$
n_{e}=\frac{4 I_{b}}{q_{e} \pi d_{b}^{2}} \sqrt{\frac{m_{e}}{2 E_{b}}}
$$

where $q_{e}$ and $m_{e}$ are the electron charge and mass, respectively, $I_{b}$ the electron-beam current, $d_{b}$ the beam diameter, and $E_{b}$ the kinetic energy of the electrons passing through the trap. Assuming a $d_{b}=100 \mu \mathrm{m}$ beam diameter, a $1 \mathrm{keV}, 100 \mathrm{~mA}$ beam corresponds to an electron density of the order $n_{e}=10^{12} \mathrm{~cm}^{-3}$. Detailed descriptions of EBITs can be found in Refs. ${ }^{41,42}$

An LCLS laser pulse of 1 ps extends about $300 \mu \mathrm{m}$ and has a diameter of about $200 \mu \mathrm{m}$. Without a monochromator, an $800 \mathrm{eV}$ pulse has roughly a $5 \mathrm{eV}$ energy spread and contains $10^{13}$ photons before entering the test cell. The number of photons that are focused to overlap with the ions in the EBIT is likely reduced by a factor of ten, which then corresponds to roughly $0.1 \mathrm{~mJ}$ of x-ray energy and a density of about $10 \mathrm{~J} \cdot \mathrm{cm}^{-3}$ delivered to the ions. By applying a monochromator the spread of the laser pulse is reduced to about $1 \mathrm{eV}$. The number of photons then decreases an order of magnitude resulting in a trap energy density of around $1 \mathrm{~J} \cdot \mathrm{cm}^{-3}$.

The structure and kinetics calculations of the iron ions were performed using the Flexible Atomic Code (FAC), a configuration-interaction program written by $\mathrm{Gu}$ for calculations of radiative and collisional processes in highly charged ions. ${ }^{40,43,44} \mathrm{FAC}$ is fully relativistic and includes some quantum electrodynamics effects, such as the Breit interaction in the zero-frequency limit, and vacuum polarization and self energy in the hydrogenic approximation. The structure of an ion with $N$ electrons is calculated by setting up and diagonalizing the Dirac-Coulomb Hamiltonian, which in atomic units can be written

$$
H=\sum_{i=1}^{N} H_{D}(i)+\sum_{i<j}^{N} \frac{1}{r_{i j}}
$$

where $H_{D}(i)$ is the Dirac Hamiltonian for electron $i$ and $r_{i j}$ denotes the distance between electrons $i$ and $j$. The configuration state functions $\Phi$ are antisymmetric sums of $N$ one-electron Dirac spinors. The radial functions are obtained from a Dirac-Fock-Slater iteration. The atomic state functions $\psi$ are then derived by summation of configuration state functions of the same symmetry. The mixing coefficients $b$ are obtained from the diagonlization of the Hamiltonian.

$$
\psi=\sum_{\nu} b_{\nu} \Phi_{\nu}
$$

The atomic state functions $\psi$ are used to calculate oscillator strengths, or $g f$ values, and radiative transition probability rates $A$, which are calculated in the single multipole approximation. From the radiative rate $A_{j i}$, between levels $j$ and $i$, the Einstein coefficient for absorption $B_{i j}$ can be calculated according to (in SI units)

$$
B_{i j}=\frac{g_{j}}{g_{i}} \frac{c^{3} h^{2}}{8 \pi \Delta E^{3}} A_{j i}
$$

where $g_{i}$ and $g_{j}$ are the statistical weights of the lower and upper levels, respectively, $c$ is the speed of light, $h$ the Planck constant, and $\Delta E$ the energy separation of the two levels (derived from Ref. ${ }^{45}$ ). The lifetime $\tau_{j}$ of an energy level $j$ equals the inverse of the sum of transition probability rates to all lower levels. 


$$
\tau_{j}=\frac{1}{\sum_{i} A_{j i}}
$$

Continuum processes are in FAC treated using the distorted-wave (DW) Born approximation, where the free orbitals are calculated taking into account the atomic structure. Nevertheless, photoionization cross sections $\sigma_{P I}$ are calculated non-resonantly and are obtained from the differential oscillator strengths (in Hartree atomic units)

$$
\sigma_{P I}=\frac{2 \pi \alpha}{g_{i}} \frac{1+\alpha^{2} \epsilon}{1+\frac{1}{2} \alpha^{2} \epsilon} \frac{d(g f)}{d E}
$$

where $\alpha$ is the fine-structure constant, $\epsilon$ the kinetic energy of the free electron, and $g_{i}$ the statistical weight of the initial bound state. ${ }^{44}$

The following constants are used when converting or calculating parameters from the FAC output files: $e=1.60217649 \times 10^{-19} \mathrm{C}, c=2.99792458 \times 10^{8} \mathrm{~ms}^{-1}, h=6.62606896 \times 10^{-34} \mathrm{Js}, m_{e}=9.10938215 \times 10^{-31} \mathrm{~kg}$.

\subsection{Atomic Structure of Ne-like Fe $\mathrm{F}^{16+}$}

The structure of sixteen-times ionized iron was calculated using configuration state functions which, in addition to the closed L-shell ground configuration, included configurations with an electron excited from the $\mathrm{K}$ shell $(n=1)$ and with one and two electrons from the L shell $(n=2)$. Excited levels include the $\mathrm{M}(n=3)$ and $\mathrm{N}$ $(n=4)$ shells. Under ordinary EBIT conditions the density is too low for two electrons of the same ion to be excited. However, it is possible that the intense photon fields from an XFEL could produce doubly excited levels in two or more steps.

The calculated transition energies, oscillator strengths, radiative transition coefficients, and lifetimes for the eight Fe XVII lines displayed in Fig. 1 are listed in Table 1. Also shown are the experimental transition energies from Brown et al., which were measured at the Livermore EBIT-II electron beam ion trap. ${ }^{46}$

Table 1. Parameters of the strong transitions in Ne-like $\mathrm{Fe}^{16+}$. Listed are theoretical transition energies, weighted oscillator strengths, Einstein transition coefficients, and upper level lifetimes. Experimental transition energies from Brown et al. ${ }^{46}$ are also included.

\begin{tabular}{cccccccc}
\hline \hline Line & Upper level & $\begin{array}{c}\Delta E(\mathrm{eV}) \\
\text { Theory }\end{array}$ & $\begin{array}{c}\Delta E(\mathrm{eV}) \\
\text { Exp. }\end{array}$ & $g f$ & $A_{j i}\left(\mathrm{~s}^{-1}\right)$ & $\tau(\mathrm{s})$ & $B_{i j}\left(\mathrm{~m}^{3} \mathrm{~s}^{-2} \mathrm{~J}^{-1}\right)$ \\
\hline $\mathrm{M} 2$ & $\left(2 \mathrm{~s}^{2} 2 \mathrm{p}_{1 / 2}^{2} 2 \mathrm{p}_{3 / 2}^{3} 3 \mathrm{~s}_{1 / 2}\right)_{2}$ & 723.6 & $725.22(4)$ & $4.65 \times 10^{-8}$ & $2.11 \times 10^{5}$ & $4.73 \times 10^{-6}$ & $3.19 \times 10^{11}$ \\
$3 \mathrm{G}$ & $\left(2 \mathrm{~s}^{2} 2 \mathrm{p}_{1 / 2}^{2} 2 \mathrm{p}_{3 / 2}^{3} 3 \mathrm{~s}_{1 / 2}\right)_{1}$ & 725.7 & $727.14(4)$ & $1.16 \times 10^{-1}$ & $8.82 \times 10^{11}$ & $1.13 \times 10^{-12}$ & $7.93 \times 10^{17}$ \\
$3 \mathrm{~F}$ & $\left(2 \mathrm{~s}^{2} 2 \mathrm{p}_{1 / 2} 2 \mathrm{p}_{3 / 2}^{4} 3 \mathrm{~s}_{1 / 2}\right)_{1}$ & 737.5 & $738.88(9)$ & $1.01 \times 10^{-1}$ & $7.93 \times 10^{11}$ & $1.26 \times 10^{-12}$ & $6.78 \times 10^{17}$ \\
$3 \mathrm{E}$ & $\left(2 \mathrm{~s}^{2} 2 \mathrm{p}_{1 / 2}^{2} 2 \mathrm{p}_{3 / 2}^{3} 3 \mathrm{~d}_{3 / 2}\right)_{1}$ & 800.9 & $802.3(3)$ & $9.79 \times 10^{-3}$ & $9.08 \times 10^{10}$ & $9.69 \times 10^{-12}$ & $6.07 \times 10^{16}$ \\
$3 \mathrm{D}$ & $\left(2 \mathrm{~s}^{2} 2 \mathrm{p}_{1 / 2}^{2} 2 \mathrm{p}_{3 / 2}^{3} 3 \mathrm{~d}_{5 / 2}\right)_{1}$ & 811.1 & $812.4(1)$ & $6.41 \times 10^{-1}$ & $6.10 \times 10^{12}$ & $1.64 \times 10^{-13}$ & $3.92 \times 10^{18}$ \\
$3 \mathrm{C}$ & $\left(2 \mathrm{~s}^{2} 2 \mathrm{p}_{1 / 2} 2 \mathrm{p}_{3 / 2}^{4} 3 \mathrm{~d}_{3 / 2}\right)_{1}$ & 825.1 & $825.79(6)$ & 2.56 & $2.52 \times 10^{13}$ & $3.97 \times 10^{-14}$ & $1.54 \times 10^{19}$ \\
$3 \mathrm{~B}$ & $\left(2 \mathrm{~s}_{1 / 2} 2 \mathrm{p}^{6} 3 \mathrm{p}_{1 / 2}\right)_{1}$ & 893.9 & $892.5(2)$ & $3.57 \times 10^{-2}$ & $4.13 \times 10^{11}$ & $1.77 \times 10^{-12}$ & $1.98 \times 10^{17}$ \\
$3 \mathrm{~A}$ & $\left(2 \mathrm{~s}_{1 / 2} 2 \mathrm{p}^{6} 3 \mathrm{p}_{3 / 2}\right)_{1}$ & 898.2 & $896.8(1)$ & $2.95 \times 10^{-1}$ & $3.44 \times 10^{12}$ & $2.78 \times 10^{-13}$ & $1.63 \times 10^{18}$ \\
\hline
\end{tabular}

The weighted absorption oscillator strengths were calculated for all the bound-bound transitions in the $\mathrm{Fe}^{16+}$ model. The oscillator strengths for transitions with energies in the $700-900 \mathrm{eV}$ interval are displayed in Fig. 2. This is the spectral range where the strong $n=2-n=3$ transitions fall within. The lines $3 \mathrm{~A}-3 \mathrm{G}$ are displayed in red. Line $3 \mathrm{C}\left(2 \mathrm{p}_{1 / 2}-3 \mathrm{~d}_{3 / 2}\right)$ has a large $g f$ value. Still, absorption from singly excited configurations to doubly excited have even larger oscillator strengths. The blue lines show $n=2-3$ transitions with an M-shell spectator electron, whereas the black lines display oscillator strengths for $n=2-3$ transitions with an N-shell spectator electron. The transitions that are excited from levels $3 \mathrm{C}$ and $3 \mathrm{D}$ are displayed in yellow and green, respectively. Even though many of these transitions have large oscillator strengths, it is not likely that they would be excited by a narrow-band FEL, since, as is clear from Fig. 2, there are few transitions in the immediate neighborhood of the $3 \mathrm{~A}-3 \mathrm{G}$ transitions. 
The differential oscillator strengths and photoionization cross sections for the bound-free transitions were furthermore calculated. The cross sections for the ground state and the eight excited levels in $\mathrm{Fe}^{16+}$ are shown in Fig. 2. The model included ionization channels from all the levels in $\mathrm{Fe}^{16+}$ to levels in the target $\mathrm{Fe}^{17+}$ ion with configurations where the seven electrons outside the filled $\mathrm{K}$ shell were either in the $\mathrm{L}$ shell or were distributed with six electrons in the $\mathrm{L}$ shell and one excited to the $\mathrm{M}$ or $\mathrm{N}$ shell. Ionization of $\mathrm{Fe}^{16+}$ then requires energies around $500 \mathrm{eV}$ to remove the $n=3$ electron, energies in excess of $1260 \mathrm{eV}$ to remove an $n=2$ electron, and around $7650 \mathrm{eV}$ to remove a K-shell electron. K-shell ionization, however, was not included in the model. It is clear from Fig. 2 that the probability of photoionization at energies around $800 \mathrm{eV}$ (i.e. where the XFEL pumps the singly excited energy levels), is quite small.
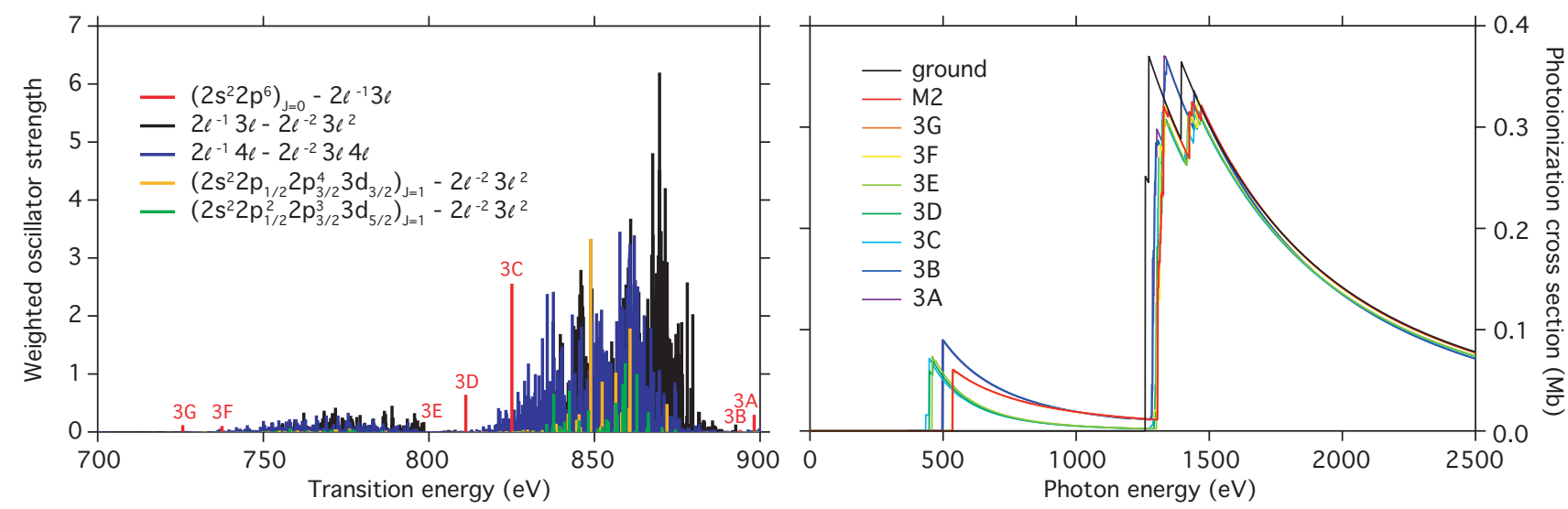

Figure 2. Left: Weighted oscillator strengths of bound-bound transitions in Fe XVII. Right: Photoionization cross sections for Fe XVII from the ground, M2, and 3A - 3G levels.

As discussed earlier, without an external radiation field the plasma in an EBIT can be considered to be in coronal equilibrium. Multistep processes are therefore of no importance and the highest charge state found in the trap is defined by the electron-beam energy. With the electron-beam energy below the threshold for direct ionization of $\mathrm{Fe}^{16+}$ (which FAC predicts to be around $1260 \mathrm{eV}$ ) only direct or multistep photoionization could create open L-shell iron ions. However, the energy of the beam only sets an upper limit of the collisionally accessible charge states. Ions with less charge can still be created, both through inelastic electron scattering and through recombination processes of ions with higher charge. Additionally, when ions are introduced into the trap with a gas injector there is always a certain amount of lower charge states present as neutral atoms or molecules are supplied to the trap continuously. The possibility of line coincidences from the sodiumlike Fe XVI spectrum with the neonlike Fe XVII spectrum should therefore be considered.

\subsection{Atomic Structure of Na-like $\mathrm{Fe}^{15+}$}

Of particular interest are the Na-like Fe XVI satellite lines to the strong Ne-like Fe XVII lines, where the analog transitions proceed with a passive spectator electron in the $n=3$ or 4 shell. For the modeling of $\mathrm{Fe}^{15+}$ only configurations with one K- or L-shell electron excited to the $\mathrm{M}$ and $\mathrm{N}$ shells were used. Levels with two electrons in the $\mathrm{M}$ or $\mathrm{N}$ shells are sensitive to autoionization as they have excitation energies above the first ionization limit around $489 \mathrm{eV}$. Electric-dipole resonance transitions that are close in energy $( \pm 10 \mathrm{eV})$ to the Ne-like Fe XVII M2, 3G, 3F, and 3D lines are listed in Table 2 (there are no E1-allowed $\mathrm{Fe}^{15+}$ resonance transitions close to $3 \mathrm{C}$ ). Theoretical transition energies, oscillator strengths, Einstein transition coefficients, and autoionization rates are presented. Some of these inner-shell transitions have recently been measured at the Livermore EBIT-I electron beam ion trap by Graf et al. ${ }^{47}$ and these experimental transition energies are also included in Table 2 for comparison.

Not all of the satellite transitions from sodiumlike iron listed in Table 2 should be observable because sevaral of the excited levels are depopulated by autoionization. The radiative decay rates $A^{r}$ are all smaller than the autoionization rates $A^{a}$ for the transitions that are close in energy to the M2 and 3G lines. No such lines were observed by Graf et al. ${ }^{47}$ However, the Fe XVI transitions close to the 3D line have radiative rates of the 
Table 2. Atomic properties of Na-like $\mathrm{Fe}^{15+}$. Theoretical transition energies, weighted oscillator strengths, radiative transition coefficients to and from the ground level $\left(2 \mathrm{~s}^{2} 2 \mathrm{p}^{6} 3 \mathrm{~s}_{1 / 2}\right)_{1 / 2}$, and autoionization rates. The experimental transition energies from Graf et al. ${ }^{47}$ are listed for comparison.

\begin{tabular}{ccccccc}
\hline \hline Upper level & $\begin{array}{c}\Delta E(\mathrm{eV}) \\
\text { Theory }\end{array}$ & $\begin{array}{c}\Delta E(\mathrm{eV}) \\
\text { Exp. }\end{array}$ & $g f$ & $A^{r}\left(\mathrm{~s}^{-1}\right)$ & $B_{i j}\left(\mathrm{~m}^{3} \mathrm{~s}^{-2} \mathrm{~J}^{-1}\right)$ & $A^{a}\left(\mathrm{~s}^{-1}\right)$ \\
\hline$\left(2 \mathrm{~s}^{2} 2 \mathrm{p}_{1 / 2} 2 \mathrm{p}_{3 / 2}^{4} 3 \mathrm{~s}_{1 / 2}^{2}\right)_{1 / 2}$ & 724.9 & & $6.36 \times 10^{-2}$ & $7.25 \times 10^{11}$ & $2.18 \times 10^{17}$ & $3.21 \times 10^{12}$ \\
$\left(2 \mathrm{~s}^{2} 2 \mathrm{p}_{1 / 2} 2 \mathrm{p}_{3 / 2}^{4} 3 \mathrm{~s}_{1 / 2} 3 \mathrm{~d}_{5 / 2}\right)_{3 / 2}$ & 803.2 & & $2.30 \times 10^{-1}$ & $1.61 \times 10^{12}$ & $7.12 \times 10^{17}$ & $7.64 \times 10^{13}$ \\
$\left(2 \mathrm{~s}^{2} 2 \mathrm{p}_{1 / 2} 2 \mathrm{p}_{3 / 2}^{4} 3 \mathrm{~s}_{1 / 2} 3 \mathrm{~d}_{3 / 2}\right)_{3 / 2}$ & 805.2 & & $3.01 \times 10^{-2}$ & $2.12 \times 10^{11}$ & $9.28 \times 10^{16}$ & $1.24 \times 10^{13}$ \\
$\left(2 \mathrm{~s}^{2} 2 \mathrm{p}_{1 / 2} 2 \mathrm{p}_{3 / 2}^{4} 3 \mathrm{p}_{3 / 2}^{2}\right)_{1 / 2}$ & 806.4 & & $1.06 \times 10^{-2}$ & $1.50 \times 10^{11}$ & $3.27 \times 10^{16}$ & $2.62 \times 10^{13}$ \\
$\left(2 \mathrm{~s}^{2} 2 \mathrm{p}_{1 / 2}^{2} 2 \mathrm{p}_{3 / 2}^{3} 3 \mathrm{p}_{1 / 2} 3 \mathrm{p}_{3 / 2}\right)_{1 / 2}$ & 808.2 & & $1.80 \times 10^{-3}$ & $2.55 \times 10^{10}$ & $5.52 \times 10^{15}$ & $4.17 \times 10^{14}$ \\
$\left(2 \mathrm{~s}^{2} 2 \mathrm{p}_{1 / 2}^{2} 2 \mathrm{p}_{3 / 2}^{3} 3 \mathrm{p}_{3 / 2}^{2}\right)_{3 / 2}$ & 810.2 & & $2.18 \times 10^{-2}$ & $1.55 \times 10^{11}$ & $6.69 \times 10^{16}$ & $3.34 \times 10^{14}$ \\
$\left(2 \mathrm{~s}^{2} 2 \mathrm{p}_{1 / 2} 2 \mathrm{p}_{3 / 2}^{4} 3 \mathrm{~s}_{1 / 2} 3 \mathrm{~d}_{5 / 2}\right)_{3 / 2}$ & 811.6 & $812.4(2)$ & 2.12 & $1.51 \times 10^{13}$ & $6.48 \times 10^{18}$ & $2.05 \times 10^{13}$ \\
$\left(2 \mathrm{~s}^{2} 2 \mathrm{p}_{1 / 2} 2 \mathrm{p}_{3 / 2}^{4} 3 \mathrm{~s}_{1 / 2} 3 \mathrm{~d}_{3 / 2}\right)_{1 / 2}$ & 814.9 & $815.1(2)$ & 1.71 & $2.46 \times 10^{13}$ & $5.21 \times 10^{18}$ & $4.61 \times 10^{12}$ \\
$\left(2 \mathrm{~s}^{2} 2 \mathrm{p}_{1 / 2} 2 \mathrm{p}_{3 / 2}^{4} 3 \mathrm{~s}_{1 / 2} 3 \mathrm{~d}_{5 / 2}\right)_{3 / 2}$ & 820.7 & $820.5(2)$ & 1.23 & $9.00 \times 10^{12}$ & $3.72 \times 10^{18}$ & $7.06 \times 10^{13}$ \\
\hline
\end{tabular}

same order of magnitude as the autoionization rates. The related parameters oscillator strengths and absorption coefficients are furthermore very large, so these transitions should easily be excited if they are pumped resonantly. As discussed by Brown et al. ${ }^{23}$ and Graf et al.,${ }^{47}$ line 3D is not appropriate for plasma diagnostics if the spectral resolution is not sufficiently high to resolve it from the Fe XVI inner-shell satellite transition. The line blend of $3 \mathrm{D}$ with the $811.6 \mathrm{eV}$ transition has been experimentally investigated by Brown et al., who measured how the apparent broadening of 3D changed as a function of the fractional $\mathrm{Fe}^{15+}$ abundance. ${ }^{23}$

It can furthermore be noted that the sodiumlike lines at $811.6 \mathrm{eV}$ and $820.7 \mathrm{eV}$, although having transitions that connect levels of different parity and a total angular momentum change of $\Delta J=1 \hbar$, the jumping electron changes $j$ by $2 \hbar$. The upper levels probably have strong configuration interaction, especially with the energetically close $\left(2 \mathrm{~s}^{2} 2 \mathrm{p}_{1 / 2}^{2} 2 \mathrm{p}_{3 / 2}^{3} 3 \mathrm{p}_{3 / 2}^{2}\right)_{3 / 2}$ level.

\subsection{The Fe XVII and Fe XVI spectra}

The laser-pumped spectra of Fe XVII and Fe XVI were modeled for photon energies centered around the excitation energies of the Fe XVII transitions M2, 3G, 3F, 3D, and 3C. Laser-beam widths of both $5 \mathrm{eV}$ and $1 \mathrm{eV}$ were used to account for full and monochromatized XFEL beams. The included atomic kinetic processes were photoexcitation, photoionization, radiative decay, autoionization, and electron capture. The spectra were modeled for optically thin plasmas, i.e. no attenuation of the laser field or resonant scattering effects were included. This should be a good approximation as an EBIT plasma has both small dimensions $\left(\sim 10^{-5} \mathrm{~cm}^{3}\right)$ and a relatively low number of ions $\left(\sim 10^{6}\right)$. The synthetic spectra are displayed in Fig. 3, where line emissivities are shown for the 3G, 3F, 3D, and 3C pumping schemes. The pumping of $\mathrm{M} 2$ is not displayed, because the magnetic quadrupole pumping does not appear sufficiently efficient in the population of the M2 level. When the level is pumped by a $5 \mathrm{eV}$ beam, however, the energetically close-lying $3 \mathrm{G}$ level gets excited and the resulting spectrum looks very similar to the $3 \mathrm{G}$ pumping scenario. It is essentially only the pumped transitions that fluoresce. Transitions that are energetically allowed, i.e. below the XFEL energy, have intensities several orders of magnitude less than the pumped transition and are therefore not likely to be important in experiments. The FAC calculated line emissivities represent the emission from a steady-state plasma and are of the order $10^{10} \mathrm{photon} /$ ion/s for the pumped transitions. However, an EBIT-XFEL plasma is highly transient since it exists approximately only during the laser-plasma interaction. Assuming an XFEL pulse length of 1 ps and a repetition rate of $100 \mathrm{~Hz}$, the emissivities get corrected by a factor of $10^{-10}$ to the intensity scales that are displayed in Fig. 3 . Note that it is the atomic line emissivities that are shown, i.e. no assumption of the plasma charge balance has been made. In a measurement the experimental conditions would likely be set to give a larger fraction of the $\mathrm{Fe}^{16+}$ ions resulting in a reduction of the Fe XVI line intensities relative the Fe XVII lines.

For comparison, the spectra of Fe XVII and Fe XVI from collisionally dominated EBIT (Gaussian excitation) and thermal (Maxwellian excitation) plasmas were also calculated. Electron-impact excitation and quenching were included for all transitions involving the ground configurations and the singly excited L-shell configurations. Also included were radiative decays from all levels, autoionization, and dielectronic recombination. No external radiation fields were now included. The EBIT spectra were modeled for an electron-beam energy of 

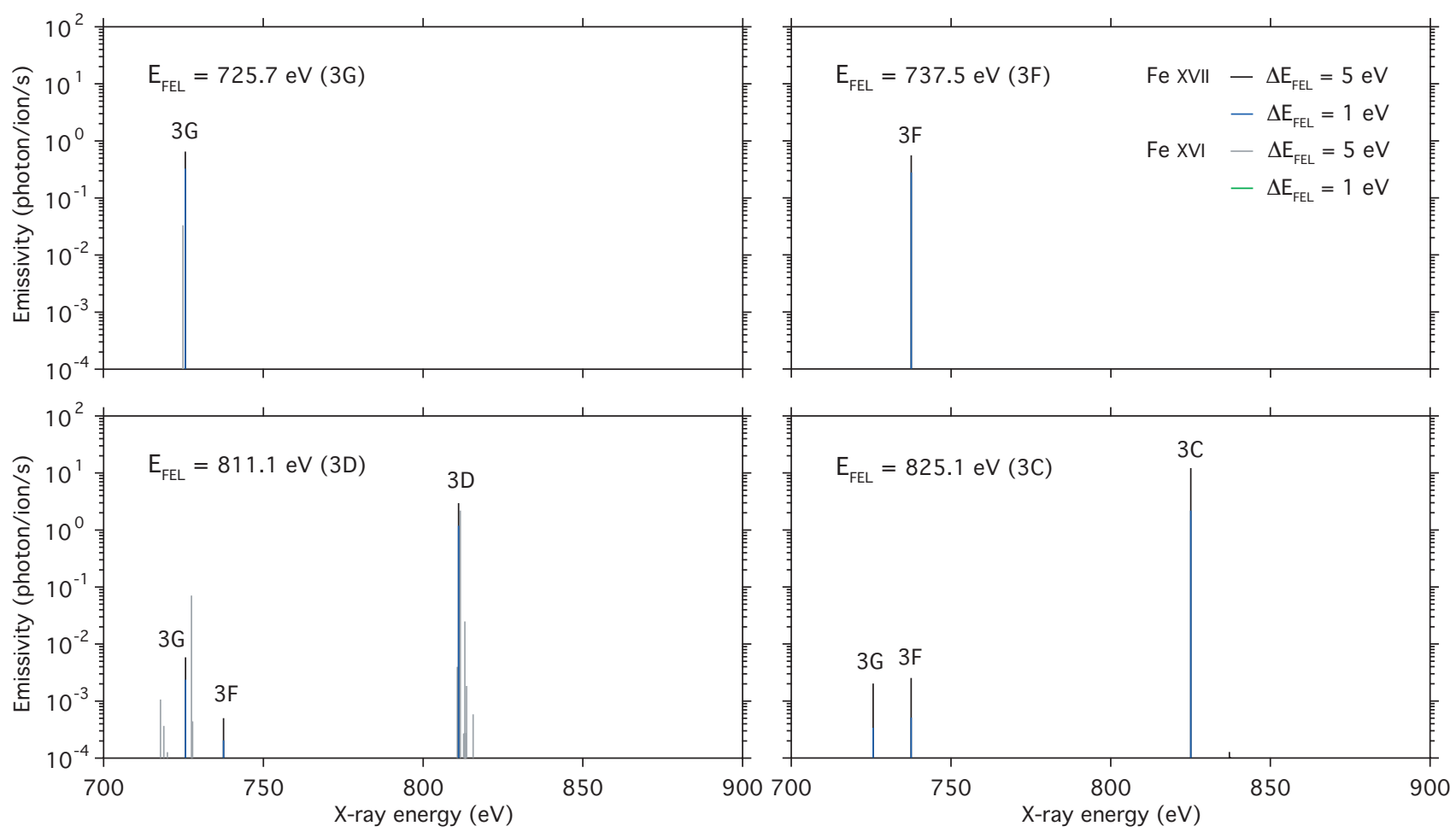

Figure 3. Theoretical spectra modeled for full free-electron laser beams ( $5 \mathrm{eV}$ width) and for XFEL beams with monochromator ( $1 \mathrm{eV}$ width). Upper left: X-ray pumping at $725.7 \mathrm{eV}$ (3G resonance). Upper right: X-ray pumping at $737.5 \mathrm{eV}$ (3F resonance). Lower left: X-ray pumping at $811.1 \mathrm{eV}$ (3D resonance). Right: X-ray pumping at $825.1 \mathrm{eV}$ (3C resonance).

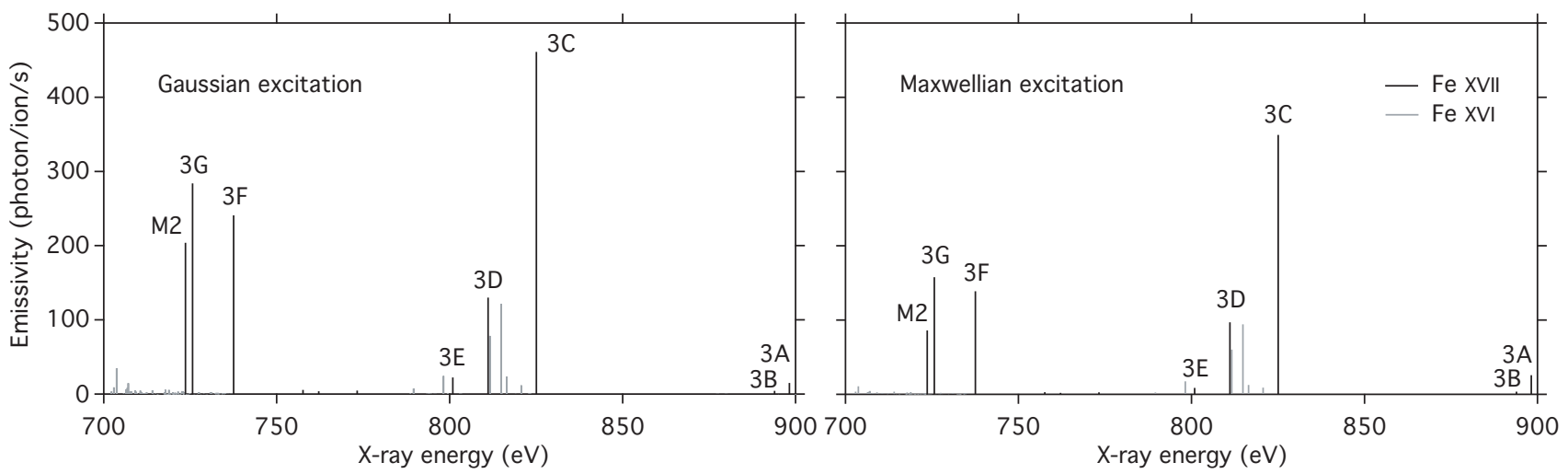

Figure 4. Collisionally excited Fe XVI and Fe XVII spectra. Left: EBIT plasma at $E_{b}=1 \mathrm{keV}$ and $n_{e}=10^{12} \mathrm{~cm}^{-3}$. Right: Thermal plasma at $T_{e}=1 \mathrm{keV}$ and $n_{e}=10^{12} \mathrm{~cm}^{-3}$.

$E_{b}=1 \mathrm{keV}$ with a Gaussian energy distribution of $30 \mathrm{eV}$ at full width at half maximum and an electron density of $1 \times 10^{12} \mathrm{~cm}^{-3} \cdot 1 \mathrm{keV}$ is well above the energy necessary to create $\mathrm{Fe}^{15+}$ and $\mathrm{Fe}^{16+}$ yet below the ionization threshold to open up the L shell. The thermal plasma spectra were calculated for the same density at an electron temperature of $T_{e}=1 \mathrm{keV}$. The electron-impact spectra are displayed in Fig. 4, where the atomic line emissivities are shown as function of transition energy.

\section{SUMMARY AND CONCLUSIONS}

The x-ray spectra of sixteen-times ionized iron have been calculated under free-electron laser excitation conditions using the FAC atomic-structure and spectral-modeling code. X-ray pumping of the diagnostically interesting 
lines M2, 3G, 3F, 3D, and 3C have been studied under conditions expected in an EBIT-XFEL plasma. The calculations, which include transition energies, oscillator strengths, photoionization cross sections, and line emissivities, indicate that the photoexcitation is very selective. This contrasts with electron-impact excitation in a regular EBIT plasma, where the well defined electron-beam energy only sets an upper limit for which transitions may be excited. Laser pumping, on the other hand, seems to transfer energy from the XFEL mostly elastically. As a consequence, lower-energy Fe XVII transitions have line intensities several orders of magnitude less than the pumped transition. Even if spectra recorded from an EBIT-XFEL plasma are integrated over several laser pulses it is therefore likely that these non-resonant transitions will be very weak. For instance, during pumping of the $3 \mathrm{D}$ transition, it would take about a minute and half for one $3 \mathrm{G}$ photon to be emitted. It is unclear why the relative intensities of lines $3 \mathrm{G}$ and $3 \mathrm{~F}$ differ for the pumping of $3 \mathrm{D}$ and $3 \mathrm{C}$.

When other ions can be expected to be present in the EBIT trap, such as the sodiumlike $\mathrm{Fe}^{15+}$ ion, line coincidences could cause the pumping of Fe XVI as well. This might be a problem for 3D excitation, where an inner-shell satellite line of Fe XVI is close in energy. As seen in Fig. 3, the line emissivity of the satellite is about the same as 3D. There are also several satellite lines in the vicinity, which could complicate measurements. Even a fractional abundance of $\mathrm{Fe}^{15+}$ that is small compared to $\mathrm{Fe}^{16+}$ could thus interfere with measurements of the 3D line. The other studied transitions in Fe XVII appear to be clear from strong line blends.

The Fe XVII and Fe XVI spectra have furthermore been modeled for collisionally dominated coronal plasmas. No external photon field was then considered so that the main line-formation processes are electron-impact excitation, recombination, and cascades. The spectra were modeled for electron densities of $n_{e}=10^{12} \mathrm{~cm}^{-3}$. The EBIT plasmas were assumed to have an $E_{b}=1 \mathrm{keV}$ Gaussian electron beam with $30 \mathrm{eV}$ full width at half maximum, and the Maxwellian plasmas an electron temperature of $T_{e}=1 \mathrm{keV}$, see Fig. 4 . The collisionally excited spectra look very different from the photopumped. All the transitions considered in this paper are now excited, with the M2 line being one of the strongest features.

As mentioned earlier, a large number of investigations of the Fe XVII spectrum has been conducted, especially during the last 15 years. To gauge the quality of the calculations presented here some comparisons are made with recent theoretical and experimental results. The calculated energy levels agree to within $1.5 \mathrm{eV}$ of the measured values by Brown et al., ${ }^{46}$ see Table 1 . The energies for 3C - 3G and M2 are all on the lower side of the experimental values, whereas the two 2s-excited levels $3 \mathrm{~A}$ and $3 \mathrm{~B}$ have slightly higher energies than measured. Although this agreement is pretty good, it may not be good enough for interpretation of astrophysical spectra, where L-shell transitions from several charge states fall within a rather narrow range, as discussed by $\mathrm{Gu}^{32,48} \mathrm{Gu}$ performed high-precision calculations by using a combined configuration-interaction and relativistic many body perturbation theory approach, which resulted in energy levels with tenths of an eV accuracy. ${ }^{32}$ Nonetheless, the present calculated energies are good enough for EBIT spectral analysis, where the emission originates from only a small number of charge states.

Several structure calculations of $\mathrm{Fe}^{16+}$ have been performed using the GRASP and SUPERSTRUCTURE codes. The radiative transition probability rates and the oscillator strengths presented here agree within $10 \%$ with these calculations. ${ }^{28,30,34,49}$ The M2 decay rate was recently measured at the Livermore EBIT-II electron beam ion trap by Crespo López-Urrutia and Beiersdorfer. ${ }^{38}$ Their value of $2.04 \times 10^{5} \mathrm{~s}$ is very close to the calculated $2.11 \times 10^{5} \mathrm{~s}$. As discussed by Crespo López-Urrutia and Beiersdorfer, there have been numerous calculations of this transition rate with results that vary widely thus impacting the quality of spectral models.

The cross sections for photoionization of $\mathrm{Fe}^{16+}$ (mainly from the ground state) have been calculated by several authors and include both relativistic and non-relativistic close-coupling and distorted-wave calculations. ${ }^{21,25,50-52}$ Comparing the result in this paper for the $\mathrm{Fe}^{16+}$ ground state, good agreement is established with the distortedwave calculations of Zhang et al. ${ }^{25}$

The atomic data used in the modeled XFEL spectra appear to be in good agreement with available experimental and theoretical data. The largest source of uncertainty in the predicted fluorescence spectra is therefore the transient nature of the pulsed plasma, which is not treated directly in the calculations. The very short duration of the laser pulse excites the levels at time scales that are of the same order as the level lifetimes. The exact relationship between these quantities may then impact the detailed level populations and corresponding emission. Additionally, the polarization of the XFEL beam could also alter the level populations. The very 
high photon intensities of over $10^{18} \mathrm{~W} \cdot \mathrm{cm}^{-2}$ achieved when the LCLS beam is focused, could furthermore affect the atomic energy level structure. ${ }^{6}$ Neither polarization, Zeeman, or Stark effects were taken into account in the spectral model. Nevertheless, the presented synthetic spectra should be a useful starting point for future photoexcitation experiments using free-electron x-ray lasers.

\section{ACKNOWLEDGEMENTS}

This work was performed under the auspices of the United States Department of Energy by Lawrence Livermore National Laboratory under Contract No. DE-AC52-07NA-27344. The author would like to acknowledge helpful discussions with P. Beiersdorfer, G. V. Brown, J. R. Crespo Lopéz-Urrutia, and A. Graf.

\section{REFERENCES}

[1] Simon, M. C., Crespo López-Urrutia, J. R., Beilmann, C., Schwarz, M., Harman, Z., Epp, S. W., Schmitt, B. L., Baumann, T. M., Behar, E., Bernitt, S., Follath, R., Ginzel, R., Keitel, C. H., Klawitter, R., Kubiček, K., Mäckel, V., Mokler, P. H., Reichardt, G., Schwarzkopf, O., and Ullrich, J., "Resonant and near-threshold photoionization cross sections of $\mathrm{Fe}^{14+}$," Phys. Rev. Lett. 105, 183001 (Oct 2010).

[2] Simon, M. C., Schwarz, M., Epp, S. W., Beilmann, C., Schmitt, B. L., Harman, Z., Baumann, T. M., Mokler, P. H., Bernitt, S., Ginzel, R., Higgins, S. G., Keitel, C. H., Klawitter, R., Kubiček, K., Mäckel, V., and Ullrich, J.and Crespo López-Urrutia, J. R., "Photoionization of $\mathrm{N}^{3+}$ and $\mathrm{Ar}^{8+}$ in an electron beam ion trap by synchrotron radiation," J. Phys. B: At. Mol. Opt. Phys. 43, 065003 (Mar 2010).

[3] Epp, S. W., Creso López-Urrutia, J. R., Simon, M. C., Baumann, T., Brenner, G., Ginzel, R., Guerassimova, N., Mäckel, V., Mokler, P. H., Schmitt, B. L., Tawara, H., and Ullrich, J., "X-ray laser spectroscopy of highly charged ions at FLASH," J. Phys. B: At. Mol. Opt. Phys. 43, 194008 (Oct 2010).

[4] Bernitt, S., Brown, G. V., Crespo López-Urrutia, J. R., Rudolph, J., Steinbrügge, R., Graf, A., Leutenegger, M., Beilmann, C., Eberle, S., Epp, S. W., Kubicek, K., Mäckel, V., Schippers, S., Schlotter, W., Simon, M. C., Träbert, E., Turner, J., Kahn, S. M., Magee, E. W., Müller, A., Porter, F. S., Rasmussen, A., Beiersdorfer, P., and Ullrich, J., "X-ray laser spectroscopy with an electron beam ion trap at the free electron laser LCLS," (2011). abstract submitted to the XXVII International Conference on Photonic, Electronic and Atomic Collisions (ICPEAC, Belfast 2011), to be published in J. Phys. Conf. Ser.

[5] Graf, A., Brown, G., Beiersdorfer, P., Clementson, J., Träbert, E., Crespo López-Urrutia, J. R., Bernitt, S., Rudolph, J., Steinbruegge, R., Eberle, S., Kubicek, K., Simon, M., Maeckel, V., Beilmann, C., Epp, S., Foucar, L., Leutenegger, M., Porter, S., Rasmussen, A., and Kahn, S., "Spectroscopic measurements of photo-excited highly charged ions of Fe and F," (2011). These proceedings.

[6] Bozek, J. D., "AMO instrumentation for the LCLS X-ray FEL," Eur. Phys. J. Special Topics 169, 129-132 (Mar 2009).

[7] Keenan, F. P. and Rose, S. J., "Spectroscopy of astrophysical plasmas in the laboratory," Astron. Geophys. 45, 6.18-6.21 (Dec 2004).

[8] Johansson, S., Derkatch, A., Donnelly, M. P., Hartman, H., Hibbert, A., Karlsson, H., Kock, M., Li, Z. S., Leckrone, D. S., Litzén, U., Lundberg, H., Mannervik, S., Norlin, L.-O., Nilsson, H., Pickering, J., Raassen, T., Rostohar, D., Royen, P., Schmitt, A., Johanning, M., M., S. C., Smith, P. L., Svanberg, S., and Wahlgren, G. M., "The FERRUM project: New f-value data for Fe II and astrophysical applications," Phys. Scr. T100, 71-80 (2002).

[9] Johansson, S., "A half-life with Fe II: tight bonds and loose ends," Phys. Scr. T134, 014013 (2009).

[10] Beiersdorfer, P., "Laboratory x-ray astrophysics," Annu. Rev. Astron. Astrophys 41, 343-390 (2003).

[11] Fournier, K. B., Finkenthal, M., Pacella, D., May, M. J., Leigheb, M., Soukhanovskii, V., and Goldstein, W. H., "Measurement of M-shell iron ionization balance in a tokamak plasma," Astrophys. J. 550, L117L120 (Mar 2001).

[12] Lepson, J. K., Beiersdorfer, P., Clementson, J., Gu, M. F., Bitter, M., Roquemore, L., Kaita, R., Cox, P. G., and Safronova, A. S., "EUV spectroscopy on NSTX," J. Phys. B: At. Mol. Opt. Phys. 43, 144018 (Jul 2010).

[13] Beiersdorfer, P., Träbert, E., and Pinnington, E. H., "Experimental transition rate of the green coronal line of Fe XIV," Astrophys. J. 587, 836-840 (Apr 2003). 
[14] Springer, P. T., Fields, D. J., Wilson, B. G., Nash, J. K., Goldstein, W. H., Iglesias, C. A., Rogers, F. J., Swenson, J. K., Chen, M. H., Bar-Shalom, A., and Stewart, R. E., "Spectroscopic absorption measurements of an iron plasma," Phys. Rev. Lett. 69, 3735-3738 (Dec 1992).

[15] Wargelin, B. J., Beiersdorfer, P., Liedahl, D. A., Kahn, S. M., and von Goeler, S., "Observation and modeling of high- $n$ iron L-shell lines from intermediate ion stages," Astrophys. J. 496, 1031-1043 (Apr 1998).

[16] Tyrén, F., "Die optischen L-spektren der Elemente Chrom bis Kobalt im extremen Ultraviolett," $Z$. Phys. 111(5-6), 314-317 (1938).

[17] Parkinson, J. H., "The analysis of a high resolution x-ray spectrum of a solar active region," Sol. Phys. 42, 183-207 (May 1975).

[18] Phillips, K. J. H., Leibacher, J. W., Wolfson, C. J., Parkinson, J. H., Fawcett, B. C., Kent, B. J., Mason, H. E., Acton, L. W., Culhane, J. L., and Gabriel, A. H., "Solar flare x-ray spectra from the Solar Maximum Mission flat crystal spectrometer," Astrophys. J. 256, 774-787 (May 1982).

[19] Saba, J. L. R., Schmelz, J. T., Bhatia, A. K., and Strong, K. T., "Fe XVII soft x-ray lines: theory and data comparisons," Astrophys. J. 510, 1064-1077 (Jan 1999).

[20] Heeter, R. F., Bailey, J. E., Cuneo, M. E., Emig, J., Foord, M. E., Springer, P. T., and Thoe, R. S., "X-ray spectroscopy of astrophysically-relevant photoionized iron plasmas at Z," in [Atomic Process in Plasmas: Twelfth Topical Conference], Mancini, R. C. and Phaneuf, R. A., eds., AIP Conference Proceedings 547, 94-108 (Nov 2000).

[21] Baliyan, K. S. and Joshi, U. C., "Photoionization cross sections for the Fe XvII ion," Bull. Astr. Soc. India 28, 311-314 (Jun 2000).

[22] Laming, J. M., Kink, I., Takacs, E., Porto, J. V., Gillaspy, J. D., Silver, E. H., Schnopper, H. W., Bandler, S. R., Brickhouse, N. S., Murray, S. S., Barbera, M., Bhatia, A. K., Doschek, G. A., Madden, N., Landis, D., Beeman, J., and Haller, E. E., "Emission-line intensity ratios in Fe XVII observed with a microcalorimeter on an electron beam ion trap," Astrophys. J. 545, L161-L164 (Dec 2000).

[23] Brown, G. V., Beiersdorfer, P., Chen, H., Chen, M. H., and Reed, K. J., "Diagnostic utility of the relative intensity of 3C to 3D in Fe XVII," Astrophys. J. 557, L75-L78 (Aug 2001).

[24] Pradhan, A. K., Nahar, S. N., and Zhang, H. L., "Unified electronic recombination of Ne-like Fe XVII: Implications for modeling x-ray plasmas," Astrophys. J. 549, L265-L268 (Mar 2001).

[25] Zhang, H. L., Nahar, S. N., and Pradhan, A. K., "Relativistic close-coupling calculations for photoionization and recombination of Ne-like Fe XVII," Phys. Rev. A 64, 032719 (Sep 2001).

[26] Beiersdorfer, P., Behar, E., Boyce, K. R., Brown, G. V., Chen, H., Gendreau, K. C., Gu, M.-F., Gygax, J., Kahn, S. M., Kelley, R. L., Porter, F. S., Stahle, C. K., and Szymkowiak, A. E., "Laboratory measurements of the relative intensity of the $3 \mathrm{~s} \rightarrow 2 \mathrm{p}$ and $3 \mathrm{~d} \rightarrow 2 \mathrm{p}$ transitions in Fe XVII," Astrophys. J. 576, L169-L172 (Sep 2002).

[27] Chen, G. X. and Pradhan, A. K., "Influence of resonances on spectral formation of x-ray lines in Fe XVII," Phys. Rev. Lett. 89, 013202 (Jul 2002).

[28] Aggarwal, K. M., Keenan, F. P., and Msezane, A. Z., "Energy levels, radiative rates, and collision strengths for transitions in Fe XVII," Astrophys. J. 144, 169-210 (Jan 2003).

[29] Nahar, S. N., Eissner, W., Chen, G.-X., and Pradhan, A. K., "Atomic data from the Iron Project. LIII. Relativistic allowed and forbidden transition probabilities for Fe XVII," Astron. Astrophys. 408, 789-801 (Sep 2003).

[30] Bhatia, A. K. and Doschek, G. A., "Atomic data and spectral line intensities for Fe XvII," At. Data Nucl. Data Tables 85, 1-45 (Sep 2003).

[31] Beiersdorfer, P., Bitter, M., von Goeler, S., and Hill, K. W., "Laboratory measurements of the Fe XVII 2p-3s and 2p-3d transitions and comparison with solar and astrophysical observations," Astrophys. J. 610, 616-623 (Jul 2004).

[32] Gu, M. F., "Wavelengths of $2 l \rightarrow 3 l^{\prime}$ transitions in L-shell ions of iron and nickel: A combined configuration interaction and many-body perturbation theory approach," Astrophys. J. Suppl. Ser. 156 (Jan 2005).

[33] Brown, G. V., Beiersdorfer, P., Chen, H., Scofield, J. H., Boyce, K. R., Kelley, R. L., Kilbourne, C. A., Porter, F. S., Gu, M. F., Kahn, S. M., and Szymkowiak, A. E., "Energy-dependent excitation cross section measurements of the diagnostics lines of Fe XVII," Phys. Rev. Lett. 96, 253201 (Jun 2006). 
[34] Loch, S. D., Pindzola, M. S., Ballance, C. P., and Griffin, D., "The effects of radiative cascades on the x-ray diagnostic lines of $\mathrm{Fe}^{16+}, "$ J. Phys. B: At. Mol. Opt. Phys. 39, 85-104 (Jan 2006).

[35] Chen, G.-X., "Converged Dirac R-matrix calculation of electron impact excitation of Fe XvII," Phys. Rev. A 76, 062708 (Dec 2007).

[36] Chen, G. X., "X-ray line ratio 3C/3D in Fe XviI," Mon. Not. R. Astron. Soc. 386, L62-L66 (May 2008).

[37] Brown, G. V., "A brief review of the intensity of lines 3C and 3D in neon-like Fe XVII," Can. J. Phys. 86, 199-208 (Jan 2008).

[38] Crespo López-Urrutia, J. R. and Beiersdorfer, P., "Measurement of the radiative decay rate of the metastable $\left(2 s^{2} 2 p_{3 / 2}^{5} 3 s_{1 / 2}\right)_{(J=2)}$ level in Fe XVII," Astrophys. J. 721, 576-581 (Sep 2010).

[39] Gillaspy, J. D., Lin, T., Tedesco, L., Tan, J. N., Pomeroy, J. M., Laming, J. M., Brickhouse, N., Chen, G.-X., and Silver, E., "Fe XVII x-ray line ratios for accurate astrophysical plasma diagnostics," Astrophys. J. 728, 132 (Feb 2011).

[40] Gu, M. F., "The Flexible Atomic Code," Can. J. Phys. 86(5), 675-689 (2008).

[41] Levine, M. A., Marrs, R. E., Henderson, J. R., Knapp, D. A., and Schneider, M. B., "The electron beam ion trap: a new instrument for atomic physics measurements," Phys. Scr. T22, 157-163 (Jan 1988).

[42] Marrs, R. E., "Milestones in EBIT spectroscopy and why it almost did not work," Can. J. Phys. 86, 11-18 (Jan 2008).

[43] Gu, M. F., "The Flexible Atomic Code," AIP Conf. Proc. 730, 127-136 (Oct 2004).

[44] Gu, M. F., FAC 1.1.1. Manual.

[45] Thorne, A., Litzén, U., and Johansson, S., [Spectrophysics - Principles and Applications], Springer-Verlag Berlin Heidelberg New York (1999).

[46] Brown, G. V., Beiersdorfer, P., Liedahl, D. A., Widmann, K., and Kahn, S. M., "Laboratory measurements and modeling of the Fe XVII spectrum," Astrophys. J. 502, 1015-2026 (Aug 1998).

[47] Graf, A., Beiersdorfer, P., Brown, G. V., and Gu, M. F., "Measurement and modeling of Na-like Fe XVI inner-shell satellites between $14.5 \AA$ and $18 \AA$," Astrophys. J. 695, 818-824 (Apr 2009).

[48] Gu, M. F., "Recent theoretical developments on atomic data for iron L-shell ions," in [X-ray diagnostics of astrophysical plasmas: Theory, Experiment, and Observation], AIP Conference Proceedings 774, 145-154 (Jun 2005).

[49] Chen, G.-X., Pradhan, A. K., and Eissner, W., "Breit-Pauli $R$-matrix calculations for electron impact excitation of Fe XVII: a benchmark study," J. Phys. B: At. Mol. Opt. Phys. 36, 453-477 (Feb 2003).

[50] Mohan, M., Le Dourneuf, M., Hibbert, A., and Burke, P. G., "Relativistic calculation for photoionization of the ground state of neonlike Fe Xvil," Phys. Rev. A 57, 3489-3492 (May 1998).

[51] Haque, N., Chakraborty, H. S., Deshmukh, P. C., Manson, S. T., Msezane, A. Z., Deb, N. C., Felfli, Z., and Gorczyca, T. W., "Relativistic effects in the photoionization of Ne-like iron," Phys. Rev. A 60, 4577-4581 (Dec 1999).

[52] Pradhan, A. K., Nahar, S. N., and Eissner, W., "The IRON project and the RMAX project: Photoionization, electron-ion recombination of Fe XVII and oscillator strengths for Fe XXII," J. Phys. Conf. Ser. 194, 022010 (Nov 2009). 